\title{
Aménagement urbain et protections juridiques spécifiques foncières
}

M. GROS

Professeur

à l'Université d'Artois Centre Ethique, Procédures et Environnement (EA 2471)

Faculté de Droit de Doual

Boulevard d'Esquerchin 59500 Douai

gros.manuel@wanadoo.fr
을 L'aménagement urbain sous toutes ses formes (travaux, constructions, aménagement, requalification, fouilles, etc.) se heurte en France à une réglementation très protectrice de l'immeuble au sens large, tant au niveau du droit de propriété (édifices menaçant ruine. immeubles sans propriétaire apparent, voies privées) que du point de la protection de certains intérêts généraux inhérents à cet immeuble (đơmanialité publique. archéologie préventive).

Mots-clés : aménagement urbain, immeuble, péril domaine public, archéologie préventive, voles privées.

\section{Urban development and specific property legal protection}

Urban development in France, regardless its nature (works, buildings, development, amendment, excavations) will face a very protective requlation regarding buildings, and this in ar extensive way, either from a property law perspective (Constructions falling in ruins, buildings without identified owner, privates roads) or from a collective rights perspective (Rights related to state property, preventive archaeology).

Key words : urban development, building, in peril, public property, preventive archaeology, private roads. 


\section{ABBRÉVIATIONS UTILISÉES}

\begin{tabular}{|c|c|c|}
\hline AJDA & & $\begin{array}{l}\text { actualité juridique de droit } \\
\text { administratif }\end{array}$ \\
\hline Bull. civ & & $\begin{array}{l}\text { Bulletin offciel des arrêts de la } \\
\text { chambre civile de la Cour de } \\
\text { cassation. }\end{array}$ \\
\hline Cass. $3^{e}$ civile & & $\begin{array}{l}\text { Cour de cassation, troisième } \\
\text { chambre civile. }\end{array}$ \\
\hline Cass. Ch. expro & & $\begin{array}{l}\text { Cour de cassation, chambre } \\
\text { des expropriations. }\end{array}$ \\
\hline $\mathrm{CH}$ & & $\begin{array}{l}\text { Code de la construction et de } \\
\text { l'habitation. }\end{array}$ \\
\hline & & Conseil d'État. \\
\hline CT & & $\begin{array}{l}\text { Code général des collectivités } \\
\text { territoriales. }\end{array}$ \\
\hline C.JEG & & $\begin{array}{l}\text { Cahiers juridiques de l'électri- } \\
\text { cité et du gaz. }\end{array}$ \\
\hline & & $\begin{array}{l}\text { Éditions techniques de droit } \\
\text { administratif. }\end{array}$ \\
\hline & & Jurisclasseur périodique. \\
\hline R. ou Rec. CE & & $\begin{array}{l}\text { Recueil Lebon des arrèts du } \\
\text { Conseil d'État. }\end{array}$ \\
\hline RD imm. & & Revue de droit immobilier. \\
\hline & & $\begin{array}{l}\text { revue du droit public et de la } \\
\text { science politique. }\end{array}$ \\
\hline & & tribunal administratif. \\
\hline & & tribunal de grande instance. \\
\hline
\end{tabular}

1

\section{Introduction}

La complexité des polices, des propriétés et réseaux divers coexistant sur un territoire à forte densité de population comme l'Europe rendent tous aménagements ou travaux divers difficiles au regard des règles de droit (droit de passage, servitudes, autorisations administratives diverses comme permis de démolir, de construire, etc.). Ces difficultés se multiplient et surtout sont spécifiques en milieu urbain ou superpositions horizontales et verticales se conjuguent.

Aussi, l'aménageur urbain, qu'il soit privé ou public, l'entreprise de travaux publics, le constructeur, voire le prospecteur rencontreront en milieu urbain quantité de problèmes juridiques liés au foncier.

Si une synthèse exhaustive s'avère impossible, il est concevable d'identifier les principaux obstacles fréquents en milieu urbain et qui rendent l'aménagement, la construction, voire la simple maitrise foncière, dignes du parcours du combattant juridique.

Sur le strict plan juridique, c'est le statut juridique de l'immeuble - voire des différentes parties d'un immeuble - au sens large (immeuble bâti, foncier non bâti, sous-sol, etc.) qui grèvera les velléités d'aménagement ou de travaux.

Pour simplifier l'aménageur urbain se heurtera non seulement au régime particulier de certains immeubles privés, mais aussi aux considérations d'intérêt général frappant certains immeubles et imposant un régime spécifique préventif ou même répressif.

Ces différents cas de figure méritent d'être examinés successivement.

\section{Les immeubles privés à statut particulier}

L'aménageur pourra se heurter au régime juridique particulier de certains immeubles privés. La spécificité de la contrainte juridique procédera en général de l'impossibilité d'agir sur l'immeuble, soit parce qu'il relève d'une police spéciale, comme celle des immeubles menaçant ruine, soit parce que son statut juridique est bloqué, dans le cas par exemple des immeubles sans propriétaire apparent, soit enfin parce que la multiplicité de ses propriétaires empêche toute solution, comme l'illustre le cas très particulier des voies privées, en particulier ouvertes à la circulation publique.

\section{1}

\section{Les immeubles menaçant ruine}

L'aménagement urbain se heurte parfois à la difficulté de faire exécuter les mesures de police en matière d'immeubles menaçant ruine.

Il existe en pratique deux types de péril visés par le code de la construction et de l'habitation : celle de péril non imminent et celle de péril imminent, la procédure de déclaration de parcelle en état d'abandon (articles L. 2243 1.2.3.4 du Code général des collectivités territoriales) relevant du long terme et ne permettant aucune réalisation d'urgence.

La procédure de péril non imminent (L, 511-2 du code la construction et de l'habitation) devant le tribunal administratif suppose une demande d'autorisation par le maire à ce tribunal de prescrire tels ou tels travaux, voire la démolition. Elle présente le double inconvénient d'une relative lenteur (quelques mois) pendant laquelle la commune doit prendre la responsabilitế d'éventuelles mesures conservatoires, et d'une crande réticence naturelle du juge administratif devant toute atteinte forcée à la propriété privée.

La procédure de péril imminent (article L. 511-3 du $\mathrm{CCH}$ ) se déroule devant le tribunal d'instance. Paradoxalement, les chances d'obtenir gain de cause sont souvent plus grandes que devant les tribunaux administratifs. En effet, la saisine du tribunal d'instance vise à la désignation d'un expert. Le juge refuse rarement une telle demande d'expertise.

L'expert peut alors rendre un rapport concluant au péril imminent et ordonnant travaux, notamment de murage, de protection du toit ou toute autre mesure utile. Le maire peut alors délivrer un arrêté de mise en demeure prévu par la loi. Si le propriétaire ne fait rien, le maire pourrait faire procéder à l'exécution forcée de ces travaux nécessaires.

Cette a paralysie n de l'immeuble est en effet la particularité la plus fréquente de l'immeuble menaçant ruine : faute de propriétaire solvable (société civile immobilière dissoute ou en liquidation), voire identifiable (indivision ou disparition des propriétaires cf. infra), celui-ci est abandonné et sert à toutes sortes d'activités gênantes (occupations, trafics divers...). Par ailleurs, de nombreux acquéreurs seraient peut-être prêts à le réhabiliter, mais aucune vente n'est réalisable, faute de vendeur. 
Pour autant le maire serait-il en droit de faire exécuter d'office les travaux de murage ou autres préconisés par l'expert? Oui, puisqu'il appartient au maire seul d'ordonner les mesures provisoires pour la sécurité, le tribunal administratif n'ayant pas à ètre saisi en application de la procédure d'urgence (CE 6/6/69 Commune d'Arcueil DA $69 n^{\circ}$ 229). Il ne peut cependant utiliser ces pouvoirs que pour une opération partielle, ne concernant pas l'ensemble de l'immeuble mais seulement un de ses éléments dangereux (Cass. Civ. ; I 23/2/88). Il est également possible de faire procéder d'office aux travaux jugés urgents par l'expert. Le coût de ceux-ci est même récupéré si possible (existence d'un propriétaire solvable) par état exécutoire au titre de l'article L. 511-4 du Code la construction et de l'habitation.

En cas d'échec de cette procédure (refus du juge de désigner expert, refus de l'expert de prescrire les travaux), le maire pourrait peut-être alors considérer qu'il n'a pas d'autre « moyen légal n (échec des procédures spécifiques du Code de la construction et de l'habitation) pour assurer ses missions au titre des articles L. 2212 du Code général des collectivités territoriales et recourrait à l'exécution forcée, par exception devenue légale. En effet, on pourrait alors naturellement penser que les conditions de l'article L. 2212-4 du CGCT sont remplies ( $\propto$ En cas de danger grave ou imminent, tels que les accidents naturels prévus au 5 de l'article L. 2212-2, le maire prescrit l'exécution des mesures de sûreté exigées par les circonstances. Il informe d'urgence le représentant de l'État dans le département et lui fait connaitre les mesures qu'il a prescrites. n)

Cet article est nécessairement séduisant, puisqu'il permet toute «mesure exigée par les circonstances $»$, mais il est très limité dans son utilisation à raison d'une jurisprudence établie : la procédure de l'article L. 2212. 4 est réservée aux dangers « naturels » et que seule la procédure spécifique de péril (imminent ou pas) permet l'exécution de travaux (CE 11 octobre 1952 Sieur Bousteleix R. 623).

La rencontre d'une " ruine ") en milieu urbain constitue ainsi un premier obstacle pour l'auteur de projet. Celui-ci devient quasi insurmontable en cas d'absence de propriétaire apparent.

\section{Les immeubles sans propriétaires apparents}

Il y a souvent en effet, notamment en banlieue parisienne des pavillons ou habitations délaissés, à l'état de quasi-abandon, créant des nuisances esthétiques, ornementales ou de sécurité.

Cette question se pose dans de nombreuses communes oú les conséquences de la Seconde Guerre mondiale, les difficultés successorales font apparaître des immeubles sans maitre. L'idée des autorités communales est alors de remédier à l'état de certaines habitations inutilisées et en état de quasi-abandon, par appropriation ou occupation, ou à tout le moins entretien par la commune.

Il n'existe aucune solution tout à la fois rapide et parfaitement régulière !

On écartera l'exercice du droit de préemption, à raison du fait que la préemption suppose une déclaration d'intention d'aliéner, ce qui suppose des propriétaires se manifestant et se faisant ainsi connaître par une vente, hypothèse exclue par définition en l'espèce.
Si la solution la plus logique parait l'expropriation en cas d'abandon manifeste, il ne faut pas écarter les hypothèses de réalisations sur biens restant à leurs propriétaires.

La procédure d'expropriation ne sera pas facile, en raison, d'une part, des problèmes de justification de I'utilité publique de l'expropriation, d'autre part, de la lourdeur et de la lenteur de la procédure lobtention d'une déclaration d'utilité publique, d'une enquête parcellaire, d'un arrêté de cessibilité, et surtout d'une obligation de publication pendant de longues années pour valoir notification des offres...).

Il existe en effet une procédure spéciale, en cas d'abandon manifeste, visant les immeubles et terrains qui ne sont manifestement plus entretenus.

La loi 89-550 du 2 août 1989 institue ainsi la procédure suivante: à la demande du conseil municipal, le maire exerce, par un procès-verbal valant arrêté municipal, la déclaration de l'état d'abandon manifeste. Ce procès-verbal est affiché pendant trois mois en mairie, est publié dans la presse locale (la loi ne disant rien, il est prudent de publier dans deux journaux locaux), et est enfin notifié aux propriétaires, et, à défaut, la notification est faite en mairie. A l'issue d'un délai, initialement de 2 ans, ramené aujourd'hui à 6 mois (loi du 2 février 1995), le maire constate la persistance de l'abandon, par un second procès-verbal. Il saisit son conseil municipal, qui décide alors le constat d'abandon manifeste. La procédure d'expropriation peut commencer,

On rappellera que dans le cadre d'une expropriation la liste des propriétaires (art. R-11-192 du Code de l'expropriation), au même titre que le plan parcellaire, est un document substantiel. Elle doit être établie d'après les documents cadastraux et les documents détenus par le conservateur des hypothèques, ou par tout autre moyen (Cass, ch. expr. 26 mars 1965, Poupard, Bull. Civ. V, n ${ }^{\circ}$ 51). Dans l'hypothèse où le propriétaire est inconnu il faut recourir aux dispositions de l'article L. 13-7 du Code de l'expropriation. En effet, cet article dispose que « si le bien exproprié n'a pu être identifié, le juge fixe l'indemnité pour le compte de qui il appartiendra ». Ambigu dans sa rédaction, cet article ouvre néanmoins une possibilité de passer outre à l'absence de propriétaire connu à condition que l'administration n'ait pas été en mesure d'effectuer les vérifications nécessaires, ce qui semble le cas en l'espèce, s'agissant souvent d'immeubles abandonnés depuis des décennies, voire depuis la Seconde Guerre mondiale.

Concrètement, il appartient de déterminer alors à qui seront notifiés les offres et mémoires. Sur ce point le législateur n'a apporté que peu d'éléments de réponse, mais on peut distinguer néanmoins deux situations.

La première concernerait le bien vacant sans maitre qui appartient alors de plein droit à l'État (anciens art. 539 et 713 du Code des domaines). Dans cette hypothèse rare, puisque le plus souvent le bien est en indivision, que des traces de propriété figurent mème si le présumé propriétaire ne peut recevoir notification d'offres, une procédure en deux temps, après transit de l'immeuble par l'Administration des domaines, est concevable. Mais il faut, le plus souvent, envisager une deuxième solution qui est de s'adresser au président du tribunal de grande instance de la situation de l'immeuble aux fins de lui demander de désigner un 
administrateur à qui seront notifiés les actes de procédures. Dans cette hypothèse il semble alors que la procédure d'expropriation pourrait se $\approx$ débloquer $»$.

Enfin et pour compléter la procédure, il sera fait mention au pied du document à publier des parties inconnues (art. R-11-28 al. 2 du Code de l'expropriation), ceci pour satisfaire aux exigences de l'article 82 du décret du 14 octobre 1955 (D. $n^{\circ}$ 55-1350) qui dispose que dans les cas exceptionnels où elle n'a pu identifier les propriétaires connus, l'Administration indique les parcelles pour lesquelles elle n'a pas été en mesure d'effectuer les vérifications nécessaires.

Cette procédure, parfaitement régulière a cependant l'inconvénient de prendre au total quelques années, et d'être extrêmement lourde, car il faut autant de procès-verbaux, et de procédure que d'immeubles et donc de dossier à gérer (recherches de propriétés au stade de l'arrêté de cessibilité...). Elle sera souvent à ce titre dissuasive pour les communes.

Une alternative à cette procédure d'expropriation pourra être d'envisager l'exécution, au titre des articles L. 511-1 et suivants du code de la construction et de l'habitation, de travaux d'office qui seraient destinés à faire cesser un péril, mais nous en avons vu les limites.

Autre solution - conservatoire et temporaire, dans le pouvoir municipal de réquisition de logements, fondée sur l'article L. 2212-2 du CGCT (pouvoirs de police générale du maire).

Mais ce pouvoir de réquisition ne peut s'effectuer qu'en cas d'urgence et à titre exceptionnel. Ainsi en pratique, hors circonstances exceptionnelles, seule la réquisition pour relogement de sans-abri est concevable.

Ainsi, la rencontre en milieu urbain d'un édifice en ruine, a fortiori s'il est sans maître constitue un véritable imbroglio juridique.

Moins irrémédiable mais tout aussi complexe est assurément le statut des voies privées ouvertes à la circulation publique.

\section{3}

\section{Les voies privées ouvertes à la circulation publique}

Les opérations d'aménagement urbain, surtout en centre des vieilles villes, révèlent parfois l'existence de voies privées ouvertes à la circulation publique qu'il est nécessaire d'aménager ou de restaurer. Il s'agit souvent de voies de desserte de résidences privées, reliées toutefois à la voie publique, mais dont l'accès est libre. La question est, le plus souvent, de savoir si un aménageur ou la commune peuvent légalement entreprendre des travaux sur cette voie affectée au seul usage des riverains, si elle peut obtenir la propriété de cette voie et si une expropriation est nécessaire.

Seul un examen préliminaire du statut juridique de la voie et de la détermination de la personne morale chargée de son entretien permettent d'envisager les cadres juridiques possibles à la réalisation de travaux.

Le statut de ces voies est le plus souvent celui « indivision forcée n) entre les propriétaires riverains (Cass. $3^{\circ}$ civile 26 novembre 1970, Morin, GP 71-1-251).

La notion de a vole privée ouverte à la circulation publique $x$, d'origine jurisprudentielle, présente un caractère de pur fait, à l'appréciation du juge du fond, qu'il s'agisse du juge administratif (à l'occasion par exemple de l'examen de la légalité d'un arrêté de police (CE 28/1/59 Ville de Versailles, DA $59 n^{\circ} 66$ ) ou du juge judiciaire (T. correctionnel de Montbéliard, 25/5/62, D. 62 page 714 ). Le critère retenu est celui du consentement du (ou des) propriétaires de la voie! Ce consentement peut naturellement être tacite, par tolérance ou soumission passive à une circulation publique. Selon une jurisprudence célèbre « une voie privée ne peut être réputée affectée à l'usage du public que si son ouverture à la circulation publique résulte du consentement, au moins tacite, des propriétaires, qu'il résulte de l'examen des pièces du dossier que des clôtures interdisaient l'accès des voies litigieuses (...) et qu'une partie au moins des propriétaires avaient entendu ainsi se prémunir contre toute ouverture à la circulation publique s (C.E 15/2/89 Commune de Mouvaux, DA 89 $n^{\circ}$ 206). A contrario, à défaut de panneaux et de clôture, il y a acceptation tacite - même des opposants au transfert dans la voirie publique - de l'ouverture à la circulation publique !

Se pose alors la question de son entretien : I'entretien d'une voie privée est évidemment à la charge des propriétaires (CE 3/12/1954 TISSERAND, DA $55 n^{\circ} 55$ ). Si une commune ne peut assumer légalement l'entretien de voies privées dont les propriétaires se réservent l'usage (CE $17 / 10 / 80$ BRAESCH, DA $80 n^{\circ} 445$ ), elle peut en toute légalité assurer en fait l'entretien d'une voie privée ouverte à la circulation publique, et ce de jurisprudence constante (CE 16/11/57 Ville de Marseille/Poro AJDA 57.458, 15/1/82 CU de Lille requête 19424, 4/5/88 Commune de Villeneuve DA 88 $n^{\circ} 402$ )

Cela procède d'une grande logique : une voie privée ouverte à la circulation est utilisée par des usagers non propriétaires et les travaux d'entretien peuvent ainsi être supportés par la collectivité. La commune a donc incontestablement en l'espèce la possibilité légale de faire ou de financer les travaux!

Mais existe-t-il un moyen de forcer les propriétaires à des travaux d'entretien?

Il existe certes des dispositions coercitives relatives à l'assainissement: en matière d'insalubrité, le maire peut toujours agir (CE 12/2/40 Wargny, R. 69) que les voies soient ou non ouvertes à la circulation publique. Mais les pouvoirs spéciaux du maire sont limités au strict domaine de l'hygiène, tels que caniveaux (CE 18/7/19 Magnie), nettoyage des trottoirs, installation de canalisations d'eau (CE 27/2/14 Kraft, R. 250), ou sont régis par des textes particuliers (loi du 22 juillet 1912 relative à l'assainissement des voles privées et codifiée aux articles L. 162-6 et suivants du code de la voirie routière).

Mais la question la plus fréquente est en fait celle de la réalisation de travaux d'aménagement et d'amélioration de la voirie contre l'avis de quelques-uns des propriétaires.

On distinguera une procédure certaine mais longue devant le juge judiciaire, de la procédure de classement dans la voirie communale.

La procédure en autorisation judiciaire de travaux sur indivision est la plus classique. Comme nous l'indiquions précédemment, le statut actuel de la voie est celui de l'indivision forcée, supposant l'unanimité absente par définition - pour la réalisation de tous travaux, même gratuitement offerts par la commune. 
Cette procédure suppose la saisine du juge judiciaire (TGI) sur l'initiative d'au moins un propriétaire (il est préférable d'en prévoir le maximum puisqu'il faut convaincre le juge : l'action doit donc être menée par tous les partisans des travaux) sur un projet de travaux d'intérêt indivisaire. Ici, le projet est facile : réfection de la voie, aménagement de celle-ci dans l'intérêt des propriétaires et des usagers confondus. La gratuité des travaux devrait convaincre sans grande difficulté le juge à ordonner lesdits travaux. Le seul inconvénient est l'opposition de quelques-uns, qui avec l'utilisation des prérogatives de procédure conduira nécessairement à plusieurs années de procédure. Le résultat (ordonnance de travaux) est certain, mais lointain, ce d'autant que sauf exécution provisoire, l'appel serait suspensif.

A défaut le classement dans la voirie communale est le seul moyen (en dehor's de la vente, de la préemption ou de l'expropriation) de transférer la propriété de la voie à la commune.

On écartera la procédure de classement d'office au titre de l'article L. 171-14 du code de la voirie routière, pour deux raisons : la première est qu'il faut un décret en CE (2 ans minimum), la seconde tient à la limite de cette procédure : celle-ci est impossible en cas d'opposition au projet de la part d'un propriétaire, la commune devant alors recourir à l'expropriation!

En fait l'hypothèse du classement d'office est prévue par l'article L. 318-3 du Code de l'urbanisme. Cet article ne s'applique qu'aux voies privées comprises dans un ensemble d'habitation, mais c'est souvent le cas. La procédure suppose une enquête publique (articles, R. 318-10 et 11 du Code de l'urbanisme), ouverte par le préfet à la demande du conseil municipal ou de propriétaires. A défaut d'accord unanime, le transfert sera prononcé par décret en Conseil d'État (cf. article L. 318-3 alinéa 3). Ce transfert vaut classement dans le domaine public de la commune et évite donc la procédure d'expropriation. (CE 3/10/69 demoiselle MASSON, DA $69 n^{\circ}$ 327.) La différence est importante puisque ce transfert prive les propriétaires - mémes opposants - de tout droit à indemnité, dès lors qu'il y a amélioration de la circulation (CE 10/2/92 Choquette et Gonzalès, requête 107113).

Ainsi, il existe de nombreuses hypothèses - les exemples cités étant les plus fréquents et spécifiques, mais ne sont pas exhaustifs - où le régime juridique de l'immeuble en milieu urbain paralyse l'action des auteurs de projet.

Mais ces exemples relevaient des droits privés, principalement du respect du droit de propriété.

D'autres " paralysies $)$, provenant cette fois de l'intérêt général, guettent l'aménageur en milieu urbain.

\section{3}

\section{Immeubles et intérêt général}

L'intérêt général d'un immeuble peut relever du fait qu'il est propriété publique et à certaines conditions appartient au domaine public. De cette domanialité publique résulte un statut juridique interdisant une appropriation privée et une protection particulière en cas d'atteinte matérielle à l'intégrité de ce domaine. Par ailleurs, indépendamment de cette domanialité publique, le sous-sol de l'immeuble peut relever d'une protection archéologique dite archéologie préventive.

\section{Domanialité publique et droits réels}

Les personnes morales de droit public (1'État, les collectivités territoriales et les établissements publicsi possèdent un domaine immobilier, réparti en deux catégories d'immeubles : ceux qui appartiennent au domaine public de la personne concernée et ceux qui relèvent de son seul domaine privé. Si ces derniers se voient appliquer le régime du droit commun, les premiers, parce qu'ils sont des dépendances du domaine public, bénéficient d'une protection particulière.

Pour simplifier, l'appartenance au domaine public, outre le cas du classement, résulte d'une réalité factuelle, constatée par le juge administratif: le bien doit être propriété d'une personne morale de droit public, affecté à un service public ou à l'usage direct du public, et spécialement aménagé à cette fin.

Répondent à ces conditions toutes les voies publiques, les ouvrages publics, les établissements des services publics, etc., mais aussi les accessoires indispensables à ces derniers. En fait, il est rare qu'une opération d'aménagement en milieu urbain ne rencontre pas une dépendance du domaine public.

Or, ce statut interdit toute cession à une personne privée, ce qui peut paralyser une opération d'aménagement ou de construction. En effet, la règle de l'inaliénabilité du domaine public interdit la cession des biens appartenant à ce domaine. Certes, une loi du 5 janvier 1988 pour les collectivités locales, après avoir réaffirmé le principe d'inaliénabilité du domaine public des collectivités locales et de leurs établissements publics (article 13 devenu l'article L. 1311-2 du Code général des collectivités territoriales, permet une dérogation, sauf sur le domaine public viaire, par les droits réels accordés sous la forme d'un bail emphytéotique, dit " administratif $),$ mais elle ne le permet qu'" en vue de l'accomplissement, pour le compte de la collectivité territoriale, d'une mission de service public ou en vue de la réalisation d'une opération d'intérêt général relevant de sa compétence $\mathrm{n}$, comme celle du 25 juillet 1994 pour l'État, afin de favoriser la valorisation du domaine public de l'État et des établissements publics nationaux, la loi n 94-631 du 25 juillet 1994 a créé, au chapitre 19 du titre $1^{\text {er }}$ du Livre II (première partie) du Code du domaine de I'État (art. L. 34-1 à L. 34-9), une autorisation d'un type nouveau constituée de droits réels au profit des occupants du domaine, ont permis la possibilité de constituer des droits réels sur leur domaine public respectif.

Mais la constitution de droits réels, si elle constitue une avancée, ne permettait à l'aménageur par exemple d'édifier en toute sécurité un immeuble ou une construction sur une dépendance du domaine public. En théorie, l'article 552 du Code civil ( $\propto$ la propriété du sol emporte la propriété du dessus et du dessous n) devrait interdire l'appropriation des équipements par un occupant (la commune) - même s'il les a construits du propriétaire du domaine public (le syndicat mixte). En fait, l'article 552 institue une présomption de propriété, mais qui n'est pas irréfragable. Ainsi, l'article 553 du même Code civil précise que « toutes constructions, plantations et ouvrages sur un terrain ou dans l'intérieur sont présumées faits par le propriétaire à ses frais et lui appartenir, si le contraire n'est pas prouvé ». En outre, une jurisprudence, au départ implicite et aujourd'hui très claire - depuis peu-du Conseil d'État, 
permet cette appropriation. Une jurisprudence relativement ancienne (Conseil d'État 3 juillet 1959, Min des TP et SNCF, R. 423, 1/7/60 Etbs Soulat, R. 442, 4/3/91 Madame Palanque, R. tabl. 976, 27/2/95 SE à lamer/Torre, R. 109) admettait que les occupants du domaine public soient propriêtaires des installations qu'ils ont édifiés sur celui-ci lorsque l'autorisation d'occuper a été délivrée pour les besoins de leur activité et non du service public, Un récent arrêt du Conseil d'État (21 avril 1997 Ministre du Budget/Société SAGIFA, requête $147602, \mathrm{DA}^{\circ} 316$ : : Considérant que l'appropriation privative d'installations superficielles édifiées par le titulaire d'une autorisation d'occupation temporaire du domaine public n'est pas incompatible avec l'inaliénabilité de celui-ci... n) est venu en faire un principe établi. On notera, toutefois, que la propriété des constructions ou réalisations construites par le bénéficiaire de droits réels est une propriété juridiquement « temporaire »), jusqu'à l'expiration du bail. Cela signifie que ce dernier sera réellement propriétaire des équipements pendant 50 ans (ou 70 ou 99), mais qu'à l'expiration de ce bail, soit il démolira ceux-ci, soit il devra les céder au franc symbolique au propriétaire public de la dépendance. L'idée est que les « équipements $x$ seront de toute façon amortis. A défaut il y aurait contrariété au principe d'inaliénabilité du domaine public, repris par l'arrêt du CE « Ministre du Budget » du 21 avril 1997 qui rappelle que ( ... ont pu légalement prévoir que le permissionnaire demeurerait propriétaire pendant la validité de la concession des installations et constructions réalisées par lui, bien que (...) la SNCF puisse, en fin d'autorisation, accéder sans indemnité à la propriété des installations... ).

Il y a eu des progrès, mais dans certaines limites que l'aménageur doit prendre en considération.

\section{2}

\section{La détérioration du domaine public}

La domanialité publique d'un immeuble n'a pas pour seule conséquence l'inaliénabilité - à tout le moins définitive (cf. supra) de ce dernier. Elle entraîne également une protection face aux agressions de toute sorte à son intégrité.

Ces « agressions $»$, si elles concernent la voirie proprement dite (voles publiques) sont constitutives de contraventions de " petite voirie 》, si elles touchent toute autre dépendance (ouvrages, sites naturels, plages, cabines téléphoniques, etc.) sont constitutives de contraventions $\alpha$ de grande voirie $»$. II s'agit, le plus souvent, d'atteintes à l'intégrité matérielle du domaine, mais ce peut également être des agissements qui ont pour effet, ou sont susceptibles d'avoir pour effet de compromettre l'usage auquel la dépendance domaniale est légalement vouée.

Les personnes susceptibles d'être poursuivies sont en règle générale, le propriétaire du bien qui a été le vecteur de l'atteinte au domaine, et cela même s'il n'est pas en réalité à l'origine de la chaîne de causalité qui a conduit à l'atteinte au domaine. Par exemple, sera retenue la responsabilité du propriétaire d'un terrain par l'intermédiaire duquel un incendie s'est communiqué au domaine public, même si cet incendie n'a pas pris naissance sur ce terrain (CE, 27 févr. 1924, Merré : Rec. CE, p. 239 - 25 juill. 1980 , min. postes : RD publ. 1981, p. 242) ou encore la commune propriétaire d'un égout par l'intermédiaire duquel des résidus ont été déversès dans un cours d'eau, et non l'entreprise émettrice (CE, 22 mars 1961, Ville de Charleville : R. p. 204). Cela dit, dans les diverses hypothèses où le propriétaire est condamné, alors qu'il estime qu'il existe un autre auteur véritable de l'atteinte au domaine, il peut user d'une action récursoire pour obtenir le remboursement des dommages-intérêts auxquels il aura été condamné (TA Paris, 11 mai 1986, Cie parisienne de chauffage urbain : CJEG 1986, p. 361, note V. Barla).

Il existe naturellement des clauses d'exonération, comme la force majeure, entendue au sens strict et surtout la faute de l'administration assimilable à un cas de force majeure. C'est le cas lorsque les équipements du domaine public étaient défectueux. Il s'agira, par exemple, de la mauvaise conception ou du mauvais fonctionnement de barrières ou passages à niveau (CE, 25 mars 1936, Baroux : Rec. CE, p. 371. - 13 juill. 1951, SNCF: Rec. CE, p. 414. - 4 févr. 1983, min. transports d Bleusse : DA 1983, $n^{\circ}$ 109). Plus fréquemment, il y aura faute de l'administration assimilable à la force majeure lorsque l'administration a émis des instructions inadaptées, ou des informations erronées, ou dans lesquels ses agents ont commis des erreurs ou négligences diverses. Cela pourra concerner, par exemple, cas très fréquent, la fourniture de plans erronés ou incomplets (CE, 8 mars 1963, min. postes c/ Bobille : Rec. CE, p. 158. - CE, 16 févr. 1994, min. postes télécom. : RD imm. 1994, n 4, p. 645); ou un panneau de signalisation erroné (CE, 9 oct. 1981 . Nerguissian : R. p. 745). Par contre, l'auteur d'une contravention de grande voirie ne peut pas s'abriter derrière le fait d'un tiers, qu'il s'agisse d'un tiers connu (CE, 28 mars 1925, Lanoy: Rec, CE, p. 366. - 29 avr. 1966, Entreprise Coutant: Rec. CE, p. 298, - 11 juin 1975, Sté des mines de fer du nord-est : Dr. adm. 1975, n² 281. - 25 juill. 1980 , min. PTT: Dr. adm. 1980, n³34) ou inconnu (CE, 4 nov. 1955, EDF : Rec. CE, p. 524).

Une des particularités de ces infractions est que, contrairement au principe normalement applicable en matière pénale, l'administration est tenue d'exercer les poursuites lorsqu'elle constate qu'une contravention de grande voirie a été commise. (CE, sect., 23 févr. 1979, Assoc. "Les amis des chemins de ronde m: Rec. CE, p. 75, concl. A. Bacquet: D. 1979, p. 405, note M. Lombard; JCP 1980 GII, 19329, note J.-F. Davignon ; RD publ. 1979, p. 1157, note M. Waline). La seule exception à cette obligation est l'hypothèse où le refus de poursuivre peut être justifié par des motifs d'intérêt public (CE, 6 févr. 1981, Comité de défense des sites de la forêt de Fouesnant: Rec, CE, p. 746).

Le préfet, ayant constaté une contravention de grande voirie, saisit le tribunal administratif. Il peut, toutefois, se désister de son action, s'il constate la réparation du dommage. C'est le cas le pus fréquent, les aménageurs contrevenants procédant, en général, à leurs frais, à la remise en l'état du domaine, ce qui conduit au désistement.

Ce contentieux peu connu est assez fréquent en milieu urbain ou de nombreux biens appartenant à divers domaines publics (État, EDF, France Télécom, etc.), appartenant aux réseaux divers s'entrecroisent, souvent en sous-sol et sont touchés par les travaux, fouilles ou constructions réalisés.

Précisément, ces travaux ou aménagements - surtout en sous-sol - en milieu urbain, peuvent être confrontés à une autre protection, cette fois sans limite, celle de l'archéologie préventive. 


\section{L'archéologie préventive}

Les grands travaux d'aménagement - notamment urbain - dégageant d'importants vestiges archéologiques, ont entraîné la nécessité de sauver ces derniers par l'organisation de fouilles dites de "sauvetage D, entreprises dans un premier temps de façon empirique puisque aucune loi n'imposait la conduite à tenir. Ces découvertes gênaient tellement les aménageurs (retards dans le programme des travaux, modifications imposées à leurs plans) qu'lils n'hésitaient pas, parfois, à détruire les vestiges archéologiques mis au jour. Si aucune réglementation générale ne protégeait les vestiges archéologiques découverts au cours de travaux étrangers à l'archéologie, certains textes particuliers assuraient leur sauvegarde (Code de l'urbanisme et permis de construire, études d'impact, etc.).

C'est seulement en 1974 que l'État français créait l'Association pour les fouilles archéologiques nationales (AFAN), association para-administrative par un accord entre le ministère de la Cuiture et le ministère de l'Économie. Cette association était chargée, entre autres, des fouilles de sauvetage, grảce ả des crédits provenant, principalement, des aménageurs. Toutefois. le monopole des fouilles, contraire au Code des marchés publics et les tarifs élevés de l'AFAN entraînèrent une réforme législative, par le vote de la loi du 17 janvier 2001 sur l'archéologie préventive.

L'archéologie préventive a pour objet « d'assurer, à terre et sous les eaux, dans les délais appropriés, la détection, la conservation ou la sauvegarde par l'étude scientifique des éléments du patrimoine archéologique affectés ou susceptibles d'être affectés par les travaux publics ou privés concourant à l'aménagement. Elle a également pour objet l'interprétation et la diffusion des résultats obtenus ») (article 1 de la loi).

Les différentes phases de l'archéologie préventive sont le diagnostic, les opérations de fouille proprement dites, l'exploitation scientifique des résultats. « Les diagnostics et opérations de fouilles d'archéologie préventive sont confiés à un établissement public national à caractère administratif $n$ (article 4 de la loi).

Les acteurs de l'archéologie préventive sont les aménageurs publics ou privés (État, collectivités territoriales, entreprises et établissements publics ou privés), mais aussi l'État, en qualité cette fois de conciliateur des nécessités de la recherche scientifique, et de la conservation du patrimoine archéologique tout en veillant au développement économique et social ; et surtout l'opérateur matériel des fouilles, en l'espèce c'est l'établissement public national à caractère administratif chargé d'organiser et de réaliser les fouilles.

Les travaux d'archéologie préventive procèdent d'une convention entre l'établissement public et l'aménageur, convention qui règle les questions de délais d'exécution des travaux, les conditions de diverses fournitures, les conséquences d'un non-respect des délais, etc.
Le financement de l'archéologie préventive relève de redevances fiscales et subventions diverses. Les redevances d'archéologie ont la nature d'une taxe fiscale, instituée par la loi. Elles ne constituent pas le coût du service rendu à l'aménageur. C'est une participation financière de l'aménageur et non la couverture intégrale du coût des opérations de diagnostic et de fouilles. Par contre, les aménageurs sont tenus à l'obligation de versement des redevances, que ces aménageurs soient des personnes publiques ou des personnes privées. Les redevances sont dues par les personnes projetant d'exécuter des travaux soumis à autorisation préalable par application du Code de l'urbanisme et dans les divers cas mentionnés dans l'article 9 de la loi. Elles répondent à deux situations très différentes en termes de tarifs : opérations de diagnostic, d'une part, et opérations de fouilles, d'autre part.

Pour les opérations de diagnostic, le montant est calculé selon une formule unique $(R=T / 320)$. $R$ représente le montant de la redevance par mètre carré. La variable T est égale à 620 , montant indexé sur l'indice du coût de la construction), pour les opérations de fouilles, le montant de la redevance due varie selon que les fouilles sont effectuées dans des sites archéologiques dits "stratifiés » ou des sites « non stratifiés ». Un site est dit stratifié « lorsqu'il présente une accumulation sédimentaire ou une superposition de structures simples ou complexes comportant des éléments du patrimoine archéologique ». La redevance pourrait varier, selon le degré de complexité de la fouille, entre cent et huit mille francs par mètre carré.

En tout cas, l'intervention de l'archéologie préventive sur un site paralyse durant de nombreux mois toute opération d'aménagement.

\section{4 \\ Conclusion}

On constatera en conclusion que les problèmes juridiques liés au foncier, notamment en milieu urbain, qu'il soit constitué du sol, du sous-sol ou même de ce qui recouvre le sol, sont nombreux et que toute atteinte, par occupation, aménagement, fouilles, extraction ou prélèvement, est encadrée de règles juridiques spécifiques. On aurait pu évoquer également le droit spécifique minier, celui des carrières, les préoccupations et principes nouveaux du droit de l'environnement, comme les questions plus complexes encore de superposition des polices, des domaines, voire du régime juridique des tréfonds. Le choix retenu d'un examen rapide des principaux obstacles juridiques liés à l'immeuble a été dicté par un souci de synthèse des questions les plus fréquentes.

Il n'en reste pas moins que la première précaution aujourd'hui du technicien en milieu urbain intervenant sur ou sous un bien immobilier urbain, comme c'est malheureusement le cas dans bien des domaines - est d'ordre... juridique. 